\title{
Corpus callosotomy-Open and endoscopic surgical techniques
}

\author{
*Matthew D. Smyth, *Ananth K. Vellimana (D, †tEishi Asano (D, and §Sandeep Sood
}

Epilepsia, 58(Suppl. 1):73-79, 2017

doi: 10.1111/epi.13681

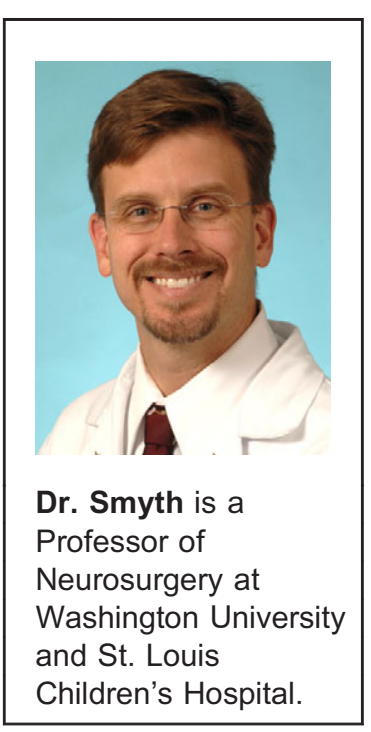

\begin{abstract}
SUMMARY
Corpus callosotomy is a palliative surgical procedure for patients with refractory epilepsy. It can be performed through an open approach via a standard craniotomy and the aid of an operating microscope, or alternatively via a mini-craniotomy with endoscope assistance. The extent of callosal disconnection performed varies according to indications and surgeon preference. In this article, we describe both open and endoscopic surgical techniques for anterior and complete corpus callosotomy.

KEY WORDS: Corpus callosotomy, Callosotomy technique, Complete callosotomy, Endoscopic callosotomy, Epilepsy surgery.
\end{abstract}

Corpus callosotomy was introduced by Van Wagenen and Herren in $1940 .^{1}$ Over time, numerous refinements in surgical technique have occurred and corpus callosotomy is now a widely accepted surgical option in appropriately selected patients. Surgical disconnection of the corpus callosum disrupts synchronization of epileptiform discharges between bilateral cerebral hemispheres and is therefore effective in reducing the severity and frequency of generalized seizures. It is a viable palliative surgical procedure for patients with medically refractory generalized seizures and partial seizures with rapid secondary generalization in the absence of an identifiable seizure focus. ${ }^{2-6}$ Generalized seizures with falls (e.g., myoclonic seizures, tonic seizures, atonic seizures) are particularly likely to respond to

Accepted September 13, 2016

*Department of Neurological Surgery, Washington University School of Medicine, St. Louis, Missouri, U.S.A.; Departments of †Pediatrics; $\$ N e u r o l o g y$; and $\S$ Neurosurgery, Wayne State University School of Medicine, Children's Hospital of Michigan, Detroit, Michigan, U.S.A.

Address correspondence to Dr. Matthew D. Smyth, One Children's Place, Suite 4s20, St. Louis, MO 63110-1077, U.S.A.. E-mail: smythm@wudosis.wustl.edu

Wiley Periodicals, Inc.

CC 2017 International League Against Epilepsy surgery. ${ }^{2,3,6}$ Although corpus callosotomy is generally well tolerated, transient or permanent postoperative neurologic deficits including hemiparesis, aphasia, mutism, akinesia, and disconnection syndromes may occur. ${ }^{2,3,6}$

The extent of callosotomy performed varies across surgical centers, and many surgeons prefer an anterior half or two thirds callosotomy sparing the splenium, as this has a lower incidence of postoperative complications. In our experience, both anterior two thirds and complete callosotomy result in improved seizure control. ${ }^{2,3}$ However, children with failed anterior two thirds callosotomy who subsequently underwent a second procedure for complete callosotomy, and those who underwent upfront complete callosotomy, experienced improvement of a broader spectrum of seizure types than those who underwent only an anterior two thirds callosotomy. ${ }^{2,3}$ Moreover, children who underwent an upfront complete callosotomy had broader seizure control than those who underwent a two-stage complete callosotomy. However, the higher potential for postoperative neurologic complications and possible unmasking of dormant seizures after a complete callosotomy must be carefully considered when deciding on the extent of callosal disconnection to be performed. ${ }^{2,3}$ 
M. D. Smyth et al.

\section{Key Points}

- Callosotomy is effective for atonic seizures and other generalized seizure types

- Anterior or complete callosotomy may be performed through a single exposure

- Complete callosotomy may provide better seizure control than anterior, but slightly increased surgical complication rates

- Endoscope-assisted callosotomy provides for a smaller incision and craniotomy and may decrease operative morbidity

Technically, both anterior and complete corpus callosotomies may be performed via an open or endoscopic approach. ${ }^{3,5}$ Although how the procedure is done is unlikely to affect the outcome, endoscopic corpus callosotomy does offer the benefit of a smaller incision, minimized brain retraction, and lower postoperative pain.

\section{Open Corpus Callosotomy}

An open corpus callosotomy involves a rectangular craniotomy approximately $8 \mathrm{~cm} \times 4 \mathrm{~cm}$, eccentric to one side along the length of the corpus callosum. Our surgical technique is described below:

\section{Positioning}

The patient is positioned supine on the operating table with the head secured using three-point pin fixation. The neck is moderately flexed and the torso is elevated 10-20 degrees above horizontal. Alternatively, the head can be positioned laterally with the right side down, to allow for gravity-assisted retraction of the frontal lobe.

\section{Preoperative adjuncts}

Preoperative magnetic resonance imaging (MRI) is coregistered to the scalp and used for frameless stereotactic navigation. Although not essential, frameless stereotactic navigation is helpful in developing trajectories to the anterior and posterior limits of the callosotomy and planning a craniotomy that avoids large bridging cortical veins. The patient is administered antibiotics, dexamethasone, and mannitol prior to skin incision. Early administration of mannitol and mild hyperventilation help with brain relaxation and facilitates the interhemispheric approach with minimal retraction.

\section{Exposure}

A trapdoor incision centered on the coronal suture, and encompassing the contralateral medial parasagittal region, is planned. Alternatively, a sigmoid or curvilinear bi-coronal incision in the region of the coronal suture may be used (Fig. 1). A limited hair shave is performed around the

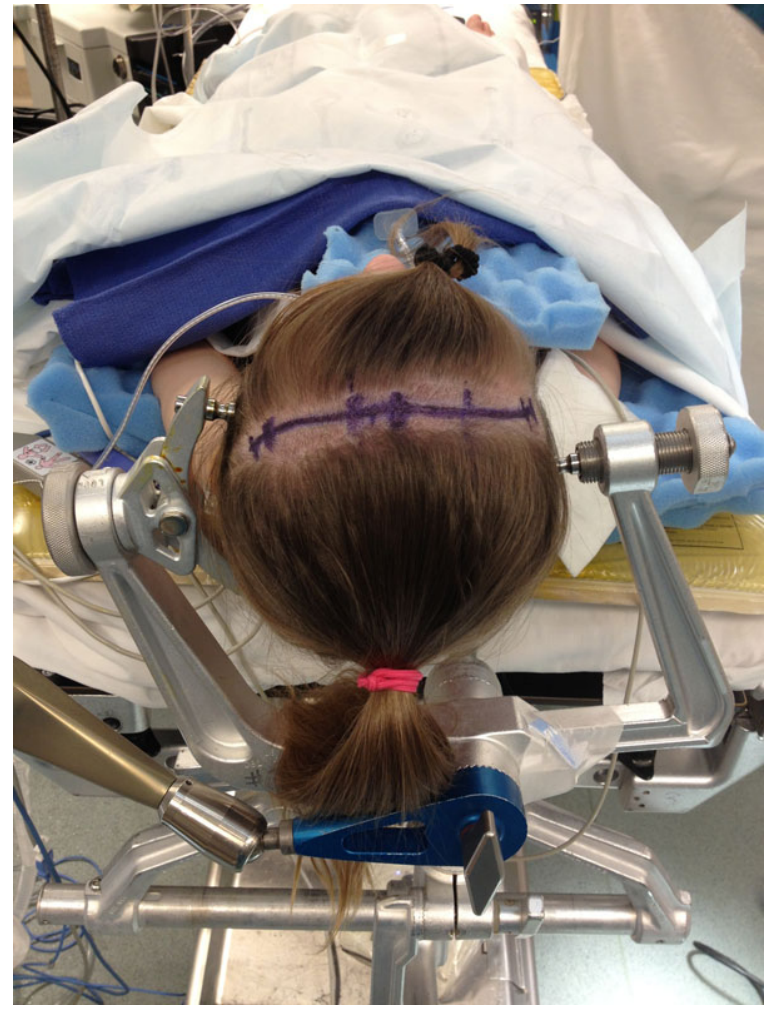

Figure I.

The patient is positioned supine with the head of bed elevated approximately 10-20 degrees above horizontal, and the neck is flexed. A curvilinear incision is marked in the region of the coronal suture.

Epilepsia (c) ILAE

planned incision. The scalp flap is created in standard fashion, and then three to six burr holes are placed for adequate epidural dissection. An $8 \mathrm{~cm}$ (anteroposterior) $\times 4 \mathrm{~cm}$

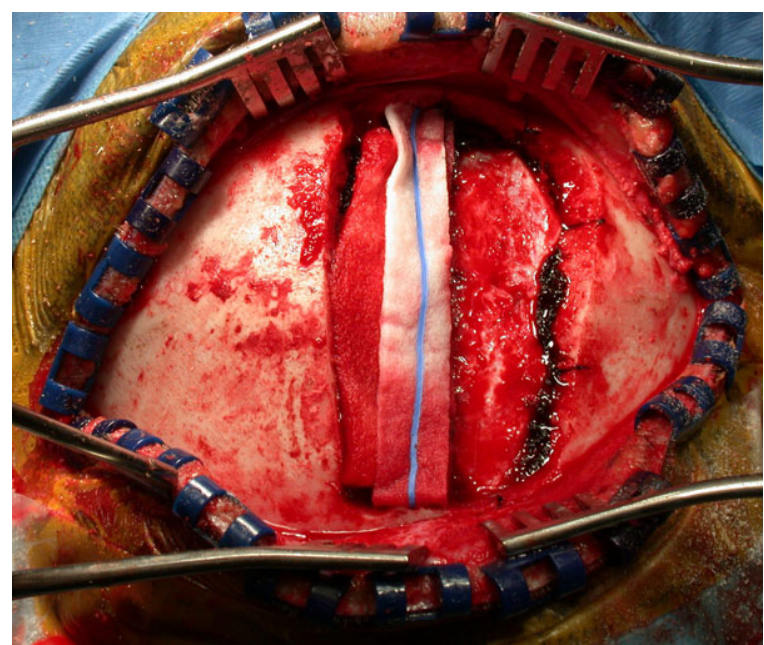

Figure 2.

A craniotomy $(\sim \mathrm{cm} \times 8 \mathrm{~cm})$ is performed straddling the coronal suture and crossing the midline. Hemostasis is achieved over the superior sagittal sinus using Surgicel, Gelfoam and cottonoids. Epilepsia (c) ILAE 


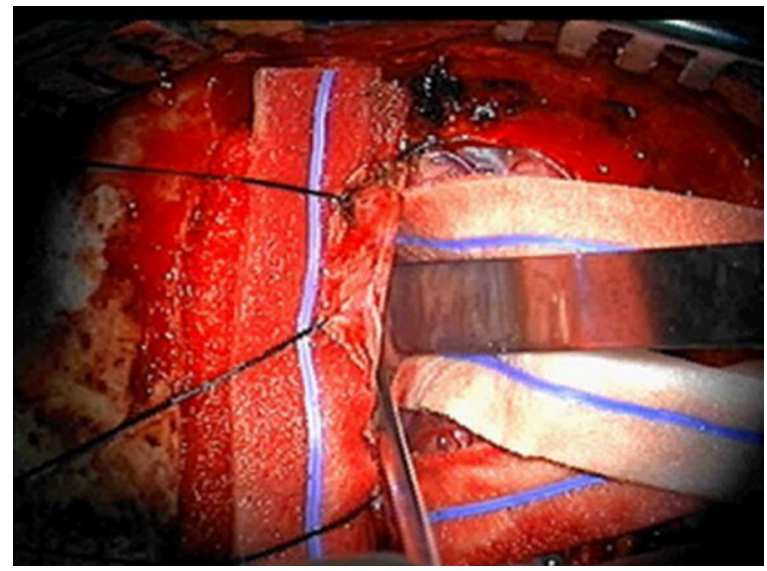

Figure 3.

A U-shaped dural opening is created and the dura is reflected toward the superior sagittal sinus to expose the interhemispheric fissure. Bridging veins posterior to the coronal suture are preserved.

Epilepsia (c) ILAE

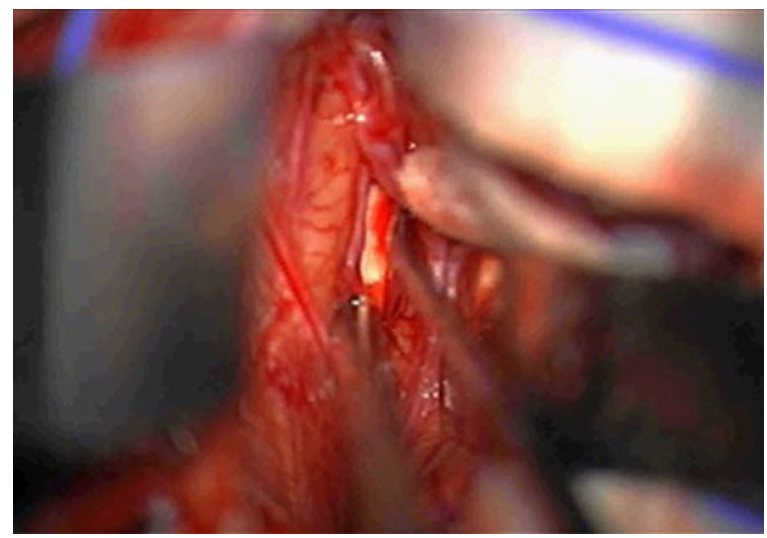

Figure 4.

Meticulous arachnoid dissection is performed in the midline avascular plane, and pericallosal arteries are separated to expose the glistening white corpus callosum.

Epilepsia C ILAE

(lateral) bone flap is created, straddling the coronal suture (Fig. 2). Bleeding over the superior sagittal sinus is usually controlled using Surgicel, Gelfoam, and cotton strips. The dura is then opened in a U-shaped fashion and reflected toward the superior sagittal sinus, until the interhemispheric fissure is visualized (Fig. 3). Care is taken to preserve all cortical veins that are at or posterior to the coronal suture.

\section{Interhemispheric approach}

Telfa or cotton strips are placed on the mesial frontal lobe to avoid cortical injury. The interhemispheric fissure is then opened and arachnoid dissection is continued to deepen the exposure. A retractor blade is positioned for gentle retraction to aid visualization, avoiding retracting the frontal lobe $>10-15 \mathrm{~mm}$. Pericallosal arteries are identified and carefully separated to each side using the midline avascular plane (Fig. 4). Further arachnoid dissection exposes the glistening white corpus callosum. The corpus callosum is then exposed along the desired extent of the callosotomy. Neuronavigation may be used to confirm midline and extent of exposure. It is preferable to complete the entire interhemispheric dissection (depending on whether an anterior two thirds or complete callosotomy is planned) prior to starting the callosotomy itself.

\section{Callosotomy}

A small callosotomy is initially created with suction and bipolar cautery under low power, and deepened to expose the midline cleft between leaves of the septum pellucidum (Fig. 5). The midline cleft is then followed anteriorly across the genu to the rostrum of the corpus callosum using suction and bipolar cautery or ultrasonic aspiration. Care is taken to preserve the ependymal lining of the ventricle to avoid risk of postoperative cerebrospinal fluid (CSF) leak and the potential for intraventricular blood. To perform a complete callosotomy, the bed is then positioned in Trendelenburg and the microscope is directed posteriorly. An intracallosal disconnection is then performed from the posterior body to the splenium. Exposure of the midline pia overlying the vein of Galen posteriorly and inferiorly confirms adequate disconnection of the splenium. Care is taken to preserve the pia to avoid injury to the internal cerebral veins and vein of Galen. Posterior callosotomy is often challenging as the angle of the splenium falls away from the surgeon. If a posterior callosotomy is later indicated, a second-stage procedure through a separate posterior incision and craniotomy provides a more direct access to the splenium.

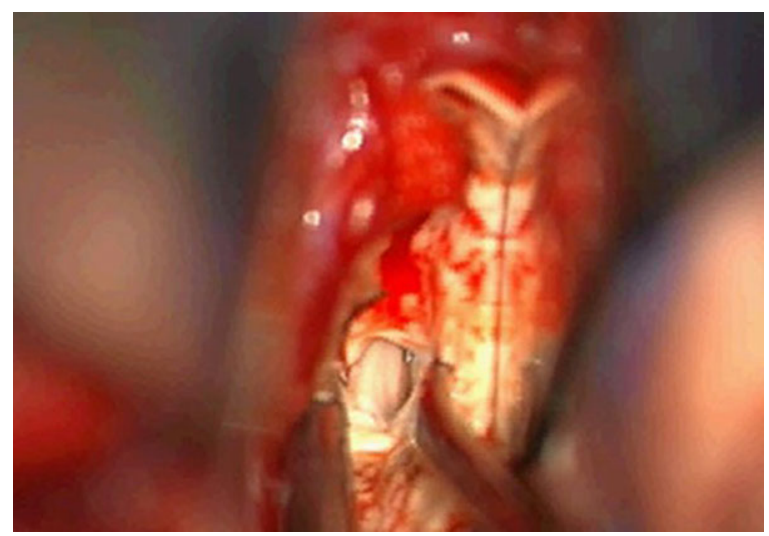

Figure 5.

After exposure of the corpus callosum along the length of the planned callosotomy, a small opening is created and this is deepened to expose midline leaves of the septum pellucidum. The callosotomy is then extended anteriorly and posteriorly to achieve the intended disconnection.

Epilepsia (C) ILAE 


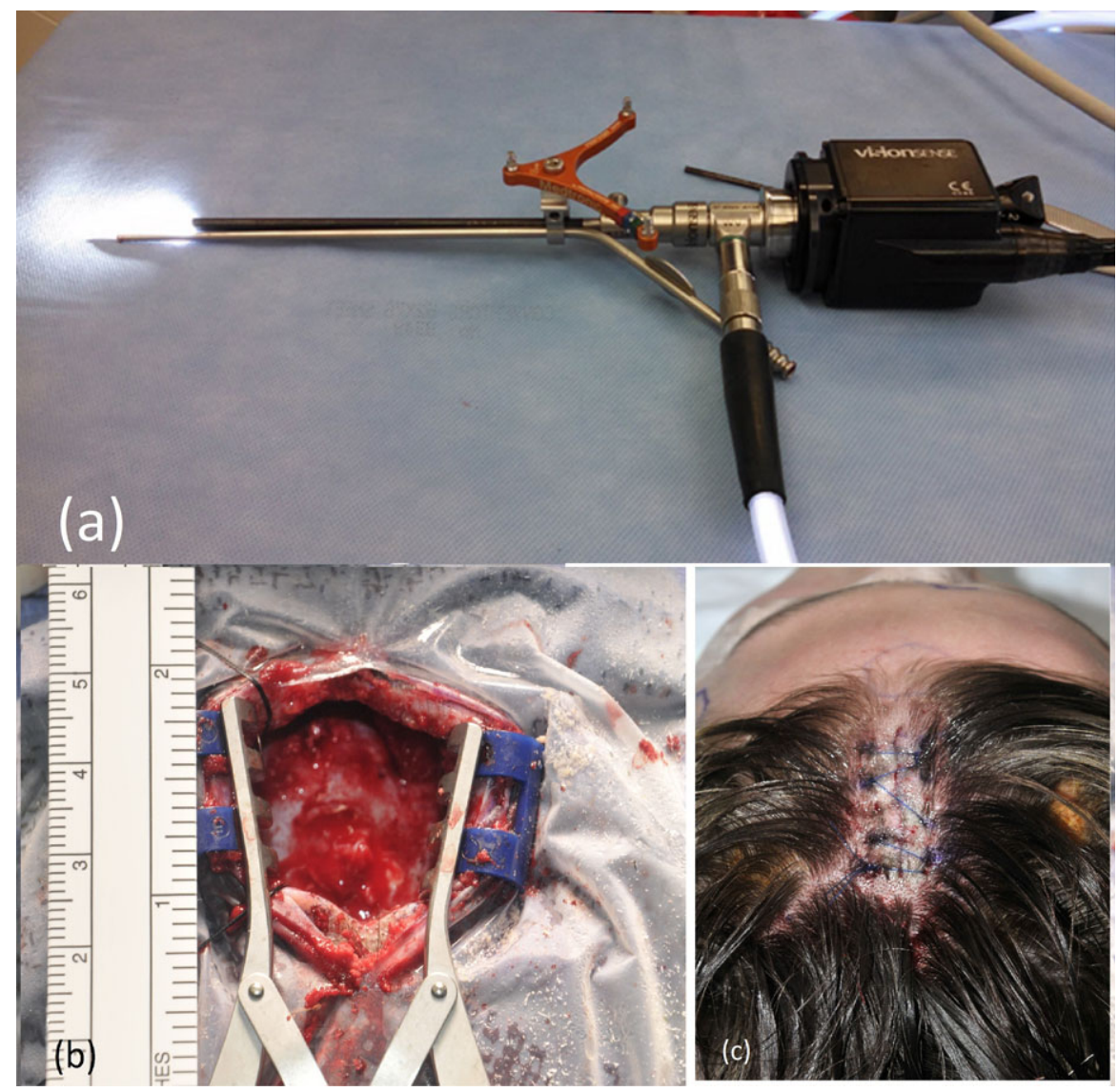

Figure 6.

(A) Endoscope with the attached suction and neuronavigation guide is held in surgeon's left hand, and bimanual dissection is performed with a second instrument held in surgeon's right hand. (B, C) The incision required to perform complete corpus callosotomy using the endoscopic method. Epilepsia (c) ILAE

\section{Closure}

After completion of the callosotomy, careful hemostasis is obtained. The dura is closed with a braided nylon suture in a water-tight fashion. The bone flap is secured to the skull with titanium plates and screws. The galea is approximated with interrupted Vicryl sutures, and the skin is closed with a running absorbable suture.

\section{Endoscopic Corpus Callosotomy}

Endoscopic corpus callosotomy aims at minimizing the size of incision and craniotomy. It was first described by Bahuleyan et al. in cadaveric dissection, utilizing anterior and posteriorly located burr holes. ${ }^{7}$ This conventional onehanded technique with instrumentation through the side channel did not offer a way of controlling hemorrhage in event of a vascular injury, or the ability to bimanually dissect, and therefore was not practical. An alternative threehanded technique, traditionally used in endoscopic transsphenoidal surgery, was recently adapted for endoscopic callosotomy by Chandra et al. ${ }^{8}$ However, in this technique, the endoscope is either held by an assistant or on a holder so that both hands of the surgeon are free to use instruments. This technique requires a larger craniotomy because the crowding of instruments necessitates more space. Moreover, because the field of surgery is larger than the view through the endoscope, it requires repeated repositioning of the endoscope, and this can be tedious and frustrating. To overcome these limitations, the author (SS) uses a clamp to attach the suction to the endoscope as shown in Figure 6A. This allows the surgeon to use the suction in the left hand and a second instrument in the right hand in a standard microsurgical fashion. Because the endoscope is attached to the suction it moves with the suction to the site of surgical dissection, thereby eliminating the need for repositioning. This two-handed technique has been used by us since 2011 and it allows safe control of hemorrhage and permits bimanual microsurgical dissection. ${ }^{5,9}$

Obscuration of vision from the instruments, lighting and depth of focus limit usefulness of conventional microscopic surgery at a depth of $>7-8 \mathrm{~cm}$ from the surface of the brain with a standard sized craniotomy. In contrast, the depth at which surgery can be performed with an endoscope is determined primarily by the length of the endoscope and can be done with a much smaller craniotomy. Using our technique, the depth to which surgery can be performed through a micro-craniotomy $(2-3 \mathrm{~cm})$ can be extended beyond the limit of the microscope. A stereotactic attachment further aids in surgical navigation. This is especially useful when a two-dimensional (2D) endoscope is used, since beyond a 
depth of $>5 \mathrm{~cm}$, surgical manipulations become difficult and require frequent reassessment of depth with neuronavigation. The author (SS) has used both 2D and 3D endoscopes for corpus callosotomy but recommends using a 3D endoscope (such as VisionSense, Philadelphia, PA, U.S.A.).

Corpus callosotomy is a low-risk operation. An open callosotomy usually does not require blood transfusion, and the risk of new deficits related to the surgical procedure such as vasospasm, retraction injury, and venous infarct was reported by us (MDS) to be about $5 \% .^{3}$ Although none of the 10 patients who had an endoscopic corpus callosotomy at our institution developed new neurologic deficits related to surgery, it would require a large number of patients to statistically demonstrate the perceived advantages of endoscopic callosotomy over standard open callosotomy. Nonetheless a smaller incision means better cosmesis, less postoperative pain, and potentially a lower risk of infection.

\section{Surgical TechniQue}

\section{Patient selection}

At the present time we do not offer endoscopic callosotomy to patients who have undergone a previous cranial operation or who have significantly distorted anatomy with a poorly formed falx and interdigitating medial hemispheres. Dissection under endoscopic vision in these patient is difficult because of adhesions and distorted anatomy.

\section{Preoperative adjuncts}

Each patient undergoes a neuronavigation MRI scan and an additional Time Resolved Imaging of Contrast KineticS sequence with magnetic resonance venography to determine the location of bridging veins. A precoronal craniotomy is planned to optimize the trajectory that allows access to the splenium and also the genu of the corpus callosum in front of the major bridging veins but behind the hairline. As with standard corpus callosotomy, antibiotic and mannitol are administered prior to incision; however, we do not use steroid intra- or postoperatively.

\section{Positioning}

In the operating room, under standard anesthesia, the patient is positioned with the trunk in slight flexion and the head in mild extension, so that the face is looking straight up and fixed in a Mayfield head holder (Integra Corp, Plainsboro, NJ, U.S.A.). This position puts the head slightly above the heart and minimizes the intracranial venous pressure. The patient is appropriately secured to allow for Trendelenburg or reverse Trendelenburg positioning during surgery. With neuronavigation registration completed, scalp electroencephalography (EEG) electrodes are placed according to the 10-20 international system of electrode placement in order to monitor EEG during the surgical procedure.

\section{Exposure}

The incision is marked about $1.5 \mathrm{~cm}$ lateral to the midline and about 1-1.5 inches in length along the sagittal plane (Fig. 6B). This region is clipped and prepped with povidone-iodine solution. Infiltration with 1:200,000 epinephrine with $2 \%$ lidocaine is used to minimize scalp bleeding. A single baby mastoid retractor with sharp teeth is used to retract the scalp, and subgaleal dissection is done without elevating the pericranium. A burr hole is made in the midline over the sagittal sinus using

\section{Figure 7.}

(A-H) Endoscopic views along the corresponding trajectories shown in (I). (See Video SI). CM,

callosomarginal artery; $\mathrm{Cg}$, cingulate gyrus; Cc, corpus callosum; Pc, pericallosal artery; ACA, anterior cerebral artery; Sp, septum pellucidum; IHF, interhemispheric fissure; Spl, splenium; Endo, endoscope; CUSA, Cavitron aspirator.

Epilepsia (c) ILAE
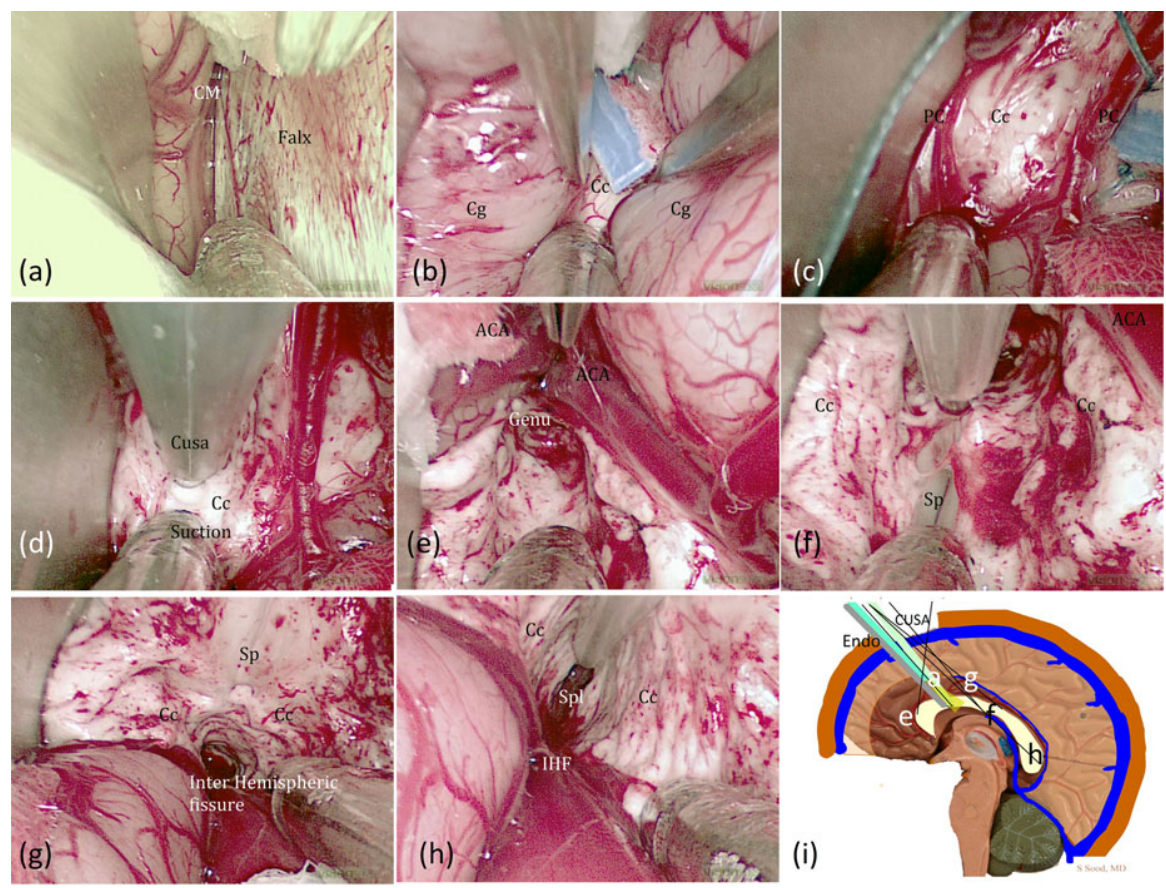


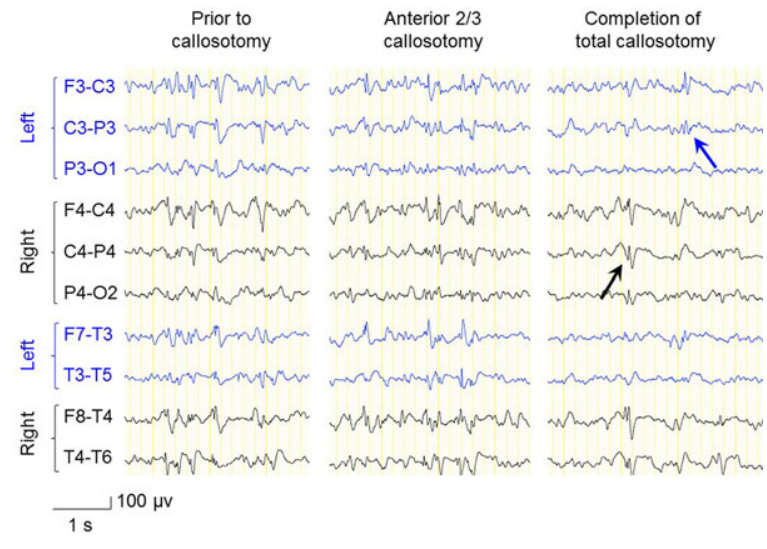

Figure 8.

Scalp EEG in an I I-year-old boy with Lennox-Gastaut syndrome. (Left) Prior to the endoscopic corpus callosotomy, scalp EEG showed frequent bisynchronous spike-wave discharges. (Middle) Upon completion of anterior two thirds corpus callosotomy, EEG still showed frequent bisynchronous spike-wave discharges. (Right) Upon completion of the total callosotomy, EEG showed occasional spike-wave discharges independently in the right and left hemispheres. Postoperatively, the patient has been free from tonic as well as astatic seizures at a 3-month follow-up period.

Epilepsia (c) ILAE

MidasRex high speed drill (Minneapolis, MN, U.S.A.). With a B1 Midas attachment, a D-shaped craniotomy is done with the straight of the $\mathrm{D}$ over the midline (Fig. 6B). The craniotomy bevel medially is outwards and the bevel anteriorly and posteriorly is inwards. This maximizes the angulation required to reach the anterior and posterior limits of resection of corpus callosum.

The dura is opened with the flap based on the sagittal sinus. Gentle lateral retraction of the brain is done to reach the lower end of the falx. A fingertip retractor may be used medially to retract the falx. Intravenous mannitol (20\%, $1 \mathrm{~g} / \mathrm{kg}$ body weight) may be used to relax the brain or, alternatively, a ventricular catheter is placed under neuronavigation guidance into the frontal horn to drain CSF.

\section{Interhemispheric exposure}

The endoscope with mounted suction is introduced alongside the falx; the cingulate gyrus in the interhemispheric fissure is dissected and the corpus callosum is exposed under endoscopic vision using the mounted suction, which aids bimanual dissection with bipolar forceps or bipolar (Fig. 7A-H; Video S1). The dissection is carried out anteriorly and posteriorly between the two pericallosal arteries to the necessary limit as delineated on the neuronavigation preoperatively. Generally, no more than $1.5-2 \mathrm{~cm}$ of exposure is necessary. Subsequently, under endoscopic vision using the mounted suction, Cavitron Ultrasonic Surgical Aspirator is used to remove the corpus callosum sequentially from anterior to posterior, while avoiding entry into the ventricle. Any bleeding can be easily controlled with a bipolar cautery.

\section{Role of intraoperative EEG recording}

Intraoperative EEG monitoring is used rather as a confirmatory diagnostic test at Children's Hospital of Michigan in Detroit. When bi-synchronous epileptiform discharges are present under general anesthesia, the evidence of desynchronization of interictal epileptiform discharges can guide the extent of callosal resection (Fig. 8). Some investigators have reported that such interhemispheric desynchronization became evident only during the resection of the posterior portion of the corpus callosum. ${ }^{10}$ Conversely, others suggest that intraoperative EEG changes alone did not effectively predict long-term seizure outcome following callosotomy ${ }^{11}$; thus the extent of callosal resection is determined by integration of multiple variables including neurologic-neuropsychological examinations, preoperative EEG abnormalities, and neuroimaging data.

\section{Closure}

Once adequate callosal resection is completed, good hemostasis is achieved, and the dura is closed in a watertight fashion with 6-0 Prolene, the bone flap is replaced and secured with plates and screws. The scalp is closed in a standard fashion in two layers. All patients are observed in the pediatric intensive care unit (ICU) overnight.

\section{Disclosure}

None of the authors has any conflict of interest to disclose. We confirm that we have read the Journal's position on issues involved in ethical publication and affirm that this report is consistent with those guidelines.

\section{REFERENCES}

1. Van Wagenen WP, Herren RY. Surgical division of the commissural pathways in the corpus callosum: relation to spread of an epileptic attack. Arch Neurol Psychiatry 1940;44:740-759.

2. Jalilian L, Limbrick DD, Steger-May K, et al. Complete versus anterior two-thirds corpus callosotomy in children: analysis of outcome. $J$ Neurosurg Pediatr 2010;6:257-266.

3. Kasasbeh AS, Smyth MD, Steger-May K, et al. Outcomes after anterior or complete corpus callosotomy in children. Neurosurgery 2014;74:17-28; discussion 28.

4. Shimizu H. Our experience with pediatric epilepsy surgery focusing on corpus callosotomy and hemispherotomy. Epilepsia 2005;46(Suppl 1):30-31.

5. Sood S, Marupudi NI, Asano E, et al. Endoscopic corpus callosotomy and hemispherotomy. J Neurosurg Pediatr 2015;16:681-686.

6. Tanriverdi T, Olivier A, Poulin N, et al. Long-term seizure outcome after corpus callosotomy: a retrospective analysis of 95 patients. $J$ Neurosurg 2009;110:332-342.

7. Bahuleyan B, Vogel TW, Robinson S, et al. Endoscopic total corpus callosotomy: cadaveric demonstration of a new approach. Pediatr Neurosurg 2011;47:455-460.

8. Chandra SP, Kurwale NS, Chibber SS, et al. Endoscopic-assisted (through a mini craniotomy) corpus callosotomy combined with anterior, hippocampal, and posterior commissurotomy in lennox-gastaut syndrome: a pilot study to establish its safety and efficacy. Neurosurgery 2016;78:743-751. 
9. Sood S, Nundkumar N, Ham SD. Interhemispheric endoscopic resection of large intraventricular and thalamic tumors. J Neurosurg Pediatr 2011;7:596-599.

10. Okumura E, Iwasaki M, Sakuraba R, et al. Time-varying inter-hemispheric coherence during corpus callosotomy. Clin Neurophysiol 2013;124:2091-2100.

11. Fiol ME, Gates JR, Mireles R, et al. Value of intraoperative EEG changes during corpus callosotomy in predicting surgical results. Epilepsia 1993;34:74-78.

\section{SUPPORTING INFORMATION}

Additional Supporting Information may be found in the online version of this article:

Video S1. Endoscopic corpus callosotomy. 